Journal of Nuclear Medicine, published on December 4, 2020 as doi:10.2967/jnumed.120.256263

PSMA-RLT proteomics/phosphoproteomics

\title{
Mechanisms of Resistance to Prostate-Specific Membrane Antigen-Targeted Radioligand Therapy in a Mouse Model of Prostate Cancer
}

Andreea D. Stuparu ${ }^{\star *}$, Joseph R. Capri2 ${ }^{\star}$, Catherine A.L. Meyer ${ }^{3 \#}$, Thuc M. Le ${ }^{3}$, Susan L. EvansAxelsson $^{4}$, Kyle Current ${ }^{3}$, Mark Lennox ${ }^{5}$, Christine E. Mona ${ }^{3,8,9}$, Wolfgang P. Fendler 6 , Jeremie Calais $^{3,8,9}$, Matthias Eiber ${ }^{7}$, Magnus Dahlbom ${ }^{3}$, Johannes Czernin ${ }^{3,8,9}$, Caius G. Radu ${ }^{3,9}$, Katharina Lückerath $3,8,9^{*}$, Roger Slavik $3^{*}$

1 Atreca Inc., South San Francisco, California, USA

2 AstraZeneca, Chemical Biology Group, Waltham, USA

3 Department of Molecular and Medical Pharmacology, David Geffen School of Medicine at University of California, Los Angeles (UCLA), California, USA

4 Department of Translational Medicine, Division of Urological Cancers, Skåne University Hospital Malmö, Lund University, Lund, Sweden

5 School of Electronics, Electrical Engineering and Computer Science, Queen's University Belfast, Belfast, Antrim, United Kingdom

6 Department of Nuclear Medicine, University of Duisburg-Essen and German Cancer Consortium (DKTK)-University Hospital Essen, Essen, Germany

7 Clinic for Nuclear Medicine, Technical University Munich, Munich, Germany

8 Department of Urology, Institute of Urologic Oncology, UCLA, California, USA.

9 Jonsson Comprehensive Cancer Center, UCLA, California, USA

*these authors contributed equally

\#Corresponding authors: Katharina Lückerath, UCLA, 650 Charles E Young Drive South, Los Angeles, CA-90095-7370, Phone: 310-206-1146, E-mail: klueckerath@mednet.ucla.edu Catherine Meyer, UCLA, 650 Charles E Young Drive South, Los Angeles, CA-90095-7370, Phone: 310-206-1146, E-mail: CAMeyer@mednet.ucla.edu 
First author: Andreea Stuparu, Atreca, Inc., 1 Tower Place, South San Francisco, CA-94080, Phone: 626-689-0315, E-mail: stuparu@gmail.com

Running title: PSMA-RLT proteomics/phosphoproteomics

Keywords: $\left.\left[{ }^{225} \mathrm{Ac}\right] \mathrm{Ac}-\mathrm{PSMA} ;{ }^{177} \mathrm{Lu}\right] \mathrm{Lu}-\mathrm{PSMA}$; prostate cancer; proteomics/phosphoproteomics; DNA damage response

Financial support: Prostate Cancer Foundation (17CHAL02, 19CHAL09), UCLA SPORE in Prostate Cancer (P50 CA092131). SLE-A: Swedish Research Council International Postdoc Grant (grant \#2015-00452). KL, WF: German Research Foundation (grants \#807454, \#807122).

Word count: 4987

Figures and tables: 5 figures; 3 supplemental figures 


\section{ABSTRACT}

Purpose: Prostate-specific membrane antigen-targeted radioligand therapy (PSMA-RLT) is effective against prostate cancer (PCa), but all patients relapse eventually. Poor understanding of the underlying resistance mechanisms represents a key barrier to development of more effective RLT. We investigate the proteome and phosphoproteome in a mouse model of PCa to identify signaling adaptations triggered by PSMA-RLT.

Experimental Design: Therapeutic efficacy of PSMA-RLT was assessed by tumor volume measurements, time to progression, and survival in C4-2 or C4-2 TP53-knockout tumor-bearing Nod scid gamma mice. Two days post-RLT, the (phospho)proteome was analyzed by mass spectrometry.

Results: PSMA-RLT significantly improved disease control in a dose-dependent manner. (Phospho)proteomic datasets revealed activation of genotoxic stress response pathways, including deregulation of DNA damage/replication stress response, TP53, androgen receptor, PI3K/AKT, and MYC signaling. C4-2 TP53-knockout tumors were less sensitive to PSMA-RLT than parental counterparts, supporting a role for TP53 in mediating RLT responsiveness.

Conclusions: We identified signaling alterations that may mediate resistance to PSMA-RLT in a PCa mouse model. Our data enable the development of rational synergistic RLT-combination therapies to improve outcomes for PCa patients. 


\section{INTRODUCTION}

Prostate specific membrane antigen-targeted radioligand therapy (PSMA-RLT) with the betaparticle emitter lutetium-177 (e.g., [ ${ }^{177}$ Lu]Lu-PSMA-617) yields responses (>50\% prostate specific antigen decline) in 57-66\% of prostate cancer (PCa) patient; however, remissions are short-lived (1). Alpha-particle emitters (e.g., actinium-225) may be superior to [177Lu]Lu-PSMA-RLT due to their higher energy radiation delivery per injected activity and increased density of ionizations responsible for DNA damage (2). Response to [ $\left.{ }^{225} \mathrm{Ac}\right] \mathrm{Ac}-\mathrm{PSMA}-\mathrm{RLT}$ have been reported in 63$76 \%$ of PCa patients (2). However, neither $\left[{ }^{225} \mathrm{Ac}\right] \mathrm{Ac}-$ nor $\left[{ }^{177} \mathrm{Lu}\right] \mathrm{Lu}-\mathrm{PSMA}-\mathrm{RLT}$ is curative and relapse occurs invariably.

lonizing radiation, as delivered by RLT, induces DNA damage. This, in turn, engages the DNA damage response/replication stress response (DDR/RSR) pathway which is a critical compensatory mechanism to cytotoxic stress in tumor cells and initiates either DNA repair or triggers cell death (3). This pathway consists of an extensive signaling cascade coordinated by the serine threonine kinases ataxia telangiectasia-mutated (ATM) and ataxia telangiectasia-andrad3-related protein (ATR) and their downstream effectors checkpoint kinases CHEK2, CHEK1 and WEE1. A similarly important mediator of DDR/RSR-induced cell cycle arrest is TP53 which induces the cyclin-dependent kinase (CDK) inhibitor p21 following cytotoxic stress.

Defects in DDR/RSR genes are common in cancer and increase the reliance on parallel DNA repair pathways (such as ATR-, ATM-, and TP53-signaling) for survival following genotoxic stress (4). In PCa, TP53 (43\% of metastatic PCa patients) together with androgen receptor (AR; $57 \%$ ), phosphatase and tensin homolog (PTEN; 35\%), ETS genes (e.g., TMPRSS2:ERG fusion, 30\%), and MYC (21\%) are among the most frequently mutated genes (5). TP53 loss-of-function has been linked to radio-resistance and tumor cell survival (6). Inactivating mutations in ATM, ATR and BRCA1/2 (which are known ATR substrates) have been linked to PCa. The mutation rates of BRCA1/2 (15\% combined), ATR $(9 \%)$ and ATM $(7 \%)$ in PCa patients are significantly higher in lethal compared with localized disease and are associated with earlier age at death and shorter 
survival time $(5,7)$. While recent clinical data have associated mutations in DDR genes with response (8) or resistance (9) to alpha-particle therapy, the roles of DDR/RSR and TP53 pathways in modulating responses to RLT in PCa have not been investigated systematically.

Here, we investigate the biological responses of a PCa mouse model to PSMA-RLT with the goal to identify actionable mechanisms potentially underlying resistance to PSMA-RLT in PCa. We profiled RLT-induced proteomic and phosphoproteomic changes in tumors to elucidate compensatory cellular stress responses and identify potential liabilities that could be exploited by novel RLT-based combination therapies. Lastly, we demonstrated that TP53 loss-of-function decreases sensitivity to PSMA-RLT in our PCa model. 


\section{METHODS}

\section{Cell Culture}

C4-2 cells were provided by Dr. G. Thalmann (Department of Urology, Inselspital Bern). C42 TP53 $\%$ cells were generated by Crispr/Cas9-knockout of tp53 using a tp53 guide RNA (GTGTAATAGCTCCTGCATGG (10)) in the lentiCRISPRv2 backbone (\# 52961, addgene; the full targeting vector was a gift of Dr. D. Nathanson, UCLA) and validated as shown in Supplemental Figure 1. Cells were maintained in Rosewell Park Memorial Institute 1640 medium/10\% fetal bovine serum at $37^{\circ} \mathrm{C}, 5 \% \mathrm{CO}_{2}$, monitored for mycoplasma contamination using the Venor GeM mycoplasma detection kit (Sigma Aldrich), and authenticated by short tandem repeat sequencing (August 2019; Laragen).

\section{Therapy Studies}

The UCLA Animal Research Committee approved all animal studies (\# 2005-090). Male, 68 weeks (wk) old Nod Scid gamma mice (UCLA Radiation Oncology Animal Core) were housed under pathogen-free conditions, food/water ad libitum, 12-12 hour light-dark cycle. Mice were observed daily to ensure animal welfare and determine if humane endpoints were reached (e.g., decreasing body condition score including severe weight loss, hunched, ruffled appearance, apathy, ulceration, tumor burden impeding with normal movement or tumor volume $\geq 3 \mathrm{~cm}^{3}$ ).

In vivo PSMA expression was verified by ${ }^{68} \mathrm{Ga}-\mathrm{PSMA}-11$ PET/CT (Supplemental Figures 1C, 2) (11).

To optimize therapeutic acitivity of $\left[{ }^{225} \mathrm{Ac}\right] \mathrm{Ac}$ - and $\left[{ }^{177} \mathrm{Lu}\right] \mathrm{Lu}-\mathrm{PSMA}-\mathrm{RLT}$ in mice with subcutaneous C4-2 tumors ( $5 \times 10^{6}$ cells, $100 \%$ matrigel), mice were randomized based on tumor volume into the following groups: Study 1, untreated, 30MBq or 120MBq [ $\left.{ }^{177} \mathrm{Lu}\right] \mathrm{Lu}-\mathrm{PSMA}-617$ (11) ( $\mathrm{n}=6 \mathrm{mice} / \mathrm{group}) ;$ Study 2 , untreated, $20 \mathrm{kBq}, 40 \mathrm{kBq}$, or $100 \mathrm{kBq}\left[{ }^{225} \mathrm{Ac}\right] \mathrm{Ac}-\mathrm{PSMA}-617$ (intravenously) (12) ( $\mathrm{n}=8 \mathrm{mice} /$ group). Therapeutic efficacy was assessed by caliper (vol = 
$\frac{1}{2}\left(\right.$ length $\times$ width $\left.^{2}\right)$; study 1 ) or computed tomography (CT; study 2$)$, time to progression to halfmaximal tumor volume (TTP), and survival.

To investigate the impact of TP53-status on RLT efficacy, mice bearing subcutaneous C4-2 or C4-2TP53\% tumors were randomized into vehicle $(0.9 \%$ saline $)$ or $\left[{ }^{177} \mathrm{Lu}\right] \mathrm{Lu}-\mathrm{PSMA}-617$ (15MBq, intravenously) groups ( $\mathrm{n}=8-10$ mice/group). Therapeutic efficacy was assessed by CT, TTP and survival.

\section{Radiochemical Synthesis}

PSMA-617 precursor $(A B X \mathrm{GmbH})$ was stored in aliquots $(1 \mathrm{mg} / \mathrm{mL})$ in $0.1 \%$ aqueous trifluoroacetic acid until use. No-carrier-added ${ }^{177} \mathrm{LuCl}_{3}$ was obtained from Spectron MRC. Actinium-225 was supplied by the Isotope Program within the Office of Nuclear Physics in the Department of Energy's Office of Science. Radiolabeling was performed at the UCLA Biomedical Cyclotron Facility as previously described, resulting in molar activities of $84 \mathrm{GBq} / \mu \mathrm{mol}$ and

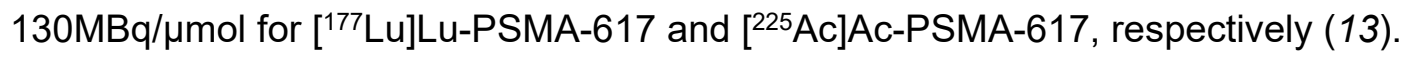

\section{Immunoblot}

TP53 (\#2527; all antibodies Cell Signaling), phospho-Chk2 (Thr68; \#2197), p21 (\#2947), phospho-Histone H2A.X (Ser139; \#9718), and $\beta$-actin (\#3700) were detected as previously published (14).

\section{PSMA expression and radiosensitivity}

PSMA expression was flow cytometrically quantified using an anti-hPSMA-APC antibody; in vitro radiosensitivity of tumor cells was assessed by propidium iodide staining (flow cytometry) and as days to confluence following irradiation (13). 


\section{Mass Spectrometry (MS)}

Tandem mass tagging and normal phase liquid chromatography-MS/MS was used to quantify the total proteome and phosphoproteome of $\mathrm{C} 4-2$ tumors $48 \mathrm{~h}$ after treatment with $40 \mathrm{kBq}$ $\left[{ }^{225}\right.$ Ac]Ac-PSMA-617 or $120 \mathrm{MBq}\left[{ }^{177}\right.$ Lu]Lu-PSMA-617 (n=3-5 tumors/group). MS data were processed using Elucidata's Polly software packages (www.elucidata.io, Elucidata, Cambridge, MA). The complete proteomic and phosphoproteomic datasets can be accessed on MassIVE (University of California San Diego; https://massive.ucsd.edu/ProteoSAFe/static/massive.jsp), identifier: MSV000086408. A detailed description is provided in the supplement.

\section{Statistics}

Statistical analysis of data was performed using GraphPad Prism 8. Statistical significance was set to $p \leq 0.05$. The Log-rank (Mantel-Cox) test was used for survival and TTP analyses. Therapeutic efficacy data were analyzed with one-way ANOVA with Bonferroni correction. For analysis of MS data, differential expression events were defined by identifying proteins/phosphopeptides with between-treatment variance significantly larger than withinreplicate variance using one-way ANOVA. Significantly altered proteins/phosphopeptides were filtered using Benjamini-Hochberg procedure at $5 \%$ false discovery rate (FDR). All statistical analyses of proteomic/phosphoproteomic MS data were performed using Python. Nucleotide pool measurements in RLT vs. control tumors were compared with two-tailed unpaired t-tests. 


\section{RESULTS}

\section{Optimizing PSMA-RLT in the C4-2 PCa model}

To identify the treatment acitivity resulting in the best anti-tumor effects without toxicity, mice were treated with varying activites of $\left[{ }^{225} \mathrm{Ac}\right] \mathrm{Ac}-$ or $\left[{ }^{177} \mathrm{Lu}\right] \mathrm{Lu}-\mathrm{PSMA}-\mathrm{RLT}$. PSMA-RLT induced significant, dose-dependent tumor shrinkage, and increased TTP and survival (Figure 1). $\left[{ }^{225} \mathrm{Ac}\right] \mathrm{Ac}-\mathrm{PSMA}-\mathrm{RLT}$ with $100 \mathrm{kBq}$ achieved the best tumor control, but mice experienced toxicity as evidenced by deteriorating mouse condition leading to a humane endpoint explaining their shorter survival. No severe weight loss was observed following ${ }^{[177}$ Lu]Lu-PSMA-RLT (Supplemental Figure 3). Comparing the efficacy of $40 \mathrm{kBq}\left[{ }^{225} \mathrm{Ac}\right] \mathrm{Ac}-\mathrm{PSMA}-\mathrm{RLT}$ vs. $30 \mathrm{MBq}$ $\left[{ }^{177} \mathrm{Lu}\right] \mathrm{Lu}-\mathrm{PSMA}-\mathrm{RLT},\left[{ }^{225} \mathrm{Ac}\right] \mathrm{Ac}-\mathrm{PSMA}-\mathrm{RLT}$ resulted in longer survival $(p=0.0019)$, but not TTP $(p=0.147)$ (the treatment activities of $30 \mathrm{MBq}$ and $40 \mathrm{kBq}$ were chosen for comparison because they most are comparable in terms of energy deposition and tumor dose; our unpublished observations).

\section{PSMA-RLT Induces DDR/RSR, Cell Cycle Arrest, and TP53 Signaling}

To investigate the molecular alterations in tumors induced by PSMA-RLT, we used global proteomics/phosphoproteomics. [ ${ }^{225} \mathrm{Ac}$ ]Ac-PSMA-RLT altered $3.3 \%$ of the proteome and $2.8 \%$ of the phosphoproteome; in $\left[{ }^{177} \mathrm{Lu}\right] \mathrm{Lu}-\mathrm{PSMA}-\mathrm{RLT}$ treated tumors, $2.5 \%$ of the total identified proteome and phosphoproteome exhibited significant differences between control and RLT groups (Figure $2 \mathrm{~A}$ ). The difference in the number of significant alterations in $\left[{ }^{225} \mathrm{Ac}\right] \mathrm{Ac}-\mathrm{vs}$. [177Lu]Lu-PSMA-RLT samples most likely reflects technical improvements of the MS methodology that were made in the time between the lutetium-177 and the actinium-225 studies, e.g., a transfer from manual to semi-automatic tissue homogenization that improved sample quality. Because of this technical development we focused on the alterations common to $\left[{ }^{225} \mathrm{Ac}\right] \mathrm{Ac}-$ and $\left[{ }^{177} \mathrm{Lu}\right] \mathrm{Lu}-$ PSMA-RLT. However, it cannot be excluded that $\left[{ }^{225} \mathrm{Ac}\right]$ Ac- vs. $\left[{ }^{177} \mathrm{Lu}\right] \mathrm{Lu}-\mathrm{PSMA}-\mathrm{RLT}$ can induce 
different alterations in the proteome/phosphoproteome due to differences in energy deposition to the surrounding tissue and the resultant differences in biological effect.

Differential regulation of DDR/RSR signaling, accompanied by cell cycle arrest, was one of the most significant alterations in PCa following PSMA-RLT (Figure 2C). RLT enhanced ATM, ATR and casein kinase 2A.1 (CSNK2A1) and suppressed CDK activity by increasing phosphorylation of ATM at Ser2996, and of known ATM, ATR and CSNK2A1 substrates (e.g., BRCA1), while decreasing the phosphorylation of CDK substrates (e.g., ribonucleotide reductase regulatory subunit M2; RRM2) (Figure 3). Upregulation of p21 and the TP53-inducible subunit RRM2B indicated enhanced TP53 activity. RLT modulated the activity of additional transcription factors involved in responses to genotoxic stress and cell cycle progression: GATA1 (15) and REST corepressor 1 (16) were upregulated, while BRCA1, MYC and E2F family members were downregulated (Figure 2D).

Supporting the notion of RLT-induced replication stress and consistent with alterations in nucleotide metabolism pathways in response to RLT (Figure 2C), nucleotide levels and expression of RRM2 and thymidine kinase 1 (TK1) were decreased by PSMA-RLT (Figure 4).

RLT deregulated AR (diminished phosphorylation of AR Ser310) and proteins regulated by or regulating $A R$, such as CSNK2A1, heat shock protein $90 A B$ (HSP90AB), and RAC-alpha serine/threonine-protein kinase (AKT1) (Figure 3) (17-19).

\section{TP53-loss renders tumors less responsive to PSMA-RLT}

Based on the proteomic/phosphoproteomic analyses (Figure 2C), and the observation that TP53 alterations are among the most common mutations in metastatic PCa, we tested if TP53status impacts RLT responsiveness in PCa. RLT resulted in excellent disease control in parental C4-2 tumors with significantly reduced tumor burden ( $p \leq 0.0376$ ), and increased TTP (NT, 27d; $R L T$, not reached; $p=0.016$ ) and survival $(N T, 38 d ; R L T$, not reached; $p=0.044)$ (Figure 5B). Mice 
with C4-2TP53-- tumors were less responsive to RLT than those with C4-2 tumors; neither tumor growth, nor TTP (NT, 14d; RLT, 30.5d; $p=0.3250$ ) or survival (NT, 21d; RLT, 42d; $p=0.3939$ ) were significantly reduced) (Figure 5C). 


\section{DISCUSSION}

Using proteomic/phosphoprotemic analysis, we identified compensatory tumor cell mechanisms that may mitigate the cytotoxic effects of RLT to confer treatment resistance, and that could be exploited for synergistic RLT-combination therapies.

Unbiased investigation of adaptive tumor cell mechanisms in response to PSMA-RLT revealed that PSMA-RLT was associated with activation of genotoxic stress response pathways and TP53-dependent cell cycle checkpoints. To our knowledge, the current study is the first systematic evaluation of the effect of RLT on DDR/RSR pathways. The relevance of DDR/RSR signaling for RLT efficacy is supported by first clinical data showing aberrations in the DDR/RSR system in 6/7 patients resistant to [225Ac]Ac-PSMA-RLT (9), and in 28 (out of 93) PCa patients responding to radium-223 treatment (8). The exact consequence of defects in or de-regulation of the DDR/RSR are likely context-dependent. DDR/RSR de-regulation can drive tumor evolution and progression due to increased genomic instability, and might contribute to mitigating the cytotoxicity from RLT to facilitate tumor cell survival. At the same time, these defects create vulnerabilities, for example by enhancing the reliance on parallel DNA repair pathways for survival following genotoxic stress (4); these pathways could be targeted to trigger excessive DNA damage and thus, cell death. In addition, high DNA damage levels and disturbance of the DDR/RSR pathway might lead to a more error-prone DNA repair, which might support tumor immunogenicity by increasing tumor mutational burden.

Clinical stage inhibitors of ATM, ATR and poly (ADP-ribose) polymerase (PARP) are synthetically lethal in DNA repair-compromising settings $(20,21)$ and have radio-sensitizing properties (3). Two studies demonstrated synergistic efficacy of thorium-227 radioimmunotherapy and inhibition of ATR or PARP $(22,23)$, and the combination of [177Lu]Lu-PSMA-RLT with the PARP inhibitor Olaparib is being investigated for the treatment of (genetically unselected) metastatic PCa (NCT03874884). [177Lu]Lu-DOTA-octreotate combined with Talazoparib 
improved disease control and survival compared to RLT alone in murine neuroendocrine tumors (24). In this context, the deregulation of ATM, ATR, CHEK2 and BRCA1 following PSMA-RLT observed in the current study supports integration of ATR (e.g., AZD6738, BAY1895344), ATM (e.g., AZD1390), and PARP (e.g., Olaparib, Talazoparib) inhibitors as radiosensitizers into RLT regimens.

TP53 is another key-effector of the DDR/RSR and mediates cell cycle arrest to allow for repair of damaged DNA. Conversely, TP53 aberrations have been linked to radio-resistance and proliferation despite severe DNA damage $(6,25)$, and TP53-knockout rendered PCa less responsive to PSMA-RLT than their TP53-wildtype counterparts (Figure 5). This finding validates our proteomic/phosphoproteomic approach to elucidate potential RLT resistance mechanisms and suggests that TP53-status is one factor impacting the outcome of PSMA-RLT. Drugging mutant TP53 to restore TP53-wildtype function has proven difficult due to the multitude of TP53 mutations with different properties (26); only one compound, APR-246, is currently being tested in clinical trials and has achieved FDA breakthrough therapy designation. However, TP53-mutant PCa exposed to RLT may particularly depend on ATM and ATR for survival (27). Future studies will investigate if inhibition of these kinases sensitizes TP53-mutant (and -wildtype) PCa to RLT.

In agreement with the activation of DDR/RSR signaling, RLT impacted nucleotide metabolism in our PCa model. Synthesis of nucleotides is essential for DNA repair as cellular nucleotide pools are limited and produced on demand (28). The two major nucleotide biosynthesis pathways are the de novo pathway, which relies on glucose and amino acids, and the salvage pathway in which preformed nucleosides are recycled (29). RLT downregulated key-effectors in de novo (RRM2, carbamoyl-phosphate synthetase 2) and salvage nucleotide biosynthesis (TK1), and decreased nucleotide levels. However, expression of deoxycytidine kinase, the second key effector in the nucleotide salvage pathway next to TK1, was not impacted by RLT. This finding suggests that deoxycytidine kinase-mediated nucleotide salvage might contribute to RLT resistance in PCa by 
enabling cells to sustain nucleoside salvage biosynthesis following RLT, thereby avoiding severe nucleotide depletion which may impair DNA repair capacity.

Further analysis of the proteome/phosphoproteome revealed targetable signaling alterations beyond canonical DDR/RSR signaling, including deregulation of AR, AKT1, MYC, and HSP90. AR transcriptional activity was enhanced following RLT, possibly due to reduced phosphorylation of AR Ser310 (30). RLT-induced AR activation may have important consequences as AR has been associated with regulation of the DDR and suppression of PSMA expression (31). Thus, AR might confer resistance to PSMA-RLT by facilitating DNA repair and reducing target expression. However, inhibition of AR signaling, which is widely used in PCa, could be exploited to upregulate PSMA expression before $\left[{ }^{68} \mathrm{Ga}\right] \mathrm{Ga}-\mathrm{PSMA}-\mathrm{PET} / \mathrm{CT}$ and PSMA-RLT with the aim to improve tumor detection, targeting and radiation dose delivery (NCT04419402, NCT04279561, NCT03977610).

The phosphatidylinositol-3-kinase (PI3K)/AKT/mammalian target of rapamycine (mTOR) pathway is a complex pathway of special interest in PCa because of its extensive crosstalk with AR signaling, and PTEN aberrations in $\sim 35 \%$ of metastatic PCa render AKT constitutively active (5). These characteristics have been associated with radio-resistance, regulation of TP53signaling, aggressive PCa phenotypes, and resistance to AR-targeted therapies (32). While $\mathrm{PISK} / \mathrm{AKT} / \mathrm{mTOR}$ inhibitors have shown promising in vitro and in vivo anti-PCa efficacy $(33,34)$, only the mTOR inhibitor Everolimus has undergone clinical testing in combination with radiotherapy (NCT01548807) (35). However, combined inhibition of AR and PI3K/AKT signaling is being explored (e.g., NCT01485861, NCT02525068) as AR inhibition can upregulate PI3K/AKT signaling (and vice versa) to maintain tumor cell survival and promote resistance. Given the radiosensitizing properties of both, AR and PI3K/AKT inhibition (36) this treatment combination might sensitize PCa to RLT while preventing the development of resistance associated with either inhibitor alone.

MYC is an important PCa driver that has been associated with numerous pro-tumorigenic 
signaling and metabolic alterations resulting in e.g., (androgen-independent) proliferation, metastasis, genomic instability, and, importantly, transition to neuroendocrine PCa $(37,38)$. Compounds reducing MYC expression or activity have shown promise for the treatment of PCa (39) and may radio-sensitize PCa (36), which supports their exploration in RLT combination therapies. Among those compounds are inhibitors of bromodomain proteins, CDKs, and HSP90. Interestingly, RLT increased the activity of HSP90AB1, a molecular chaperone for many proteins implicated in DNA repair signaling cascades, including AR (40). HSP90 is an emerging target in PCa, and its inhibition can upregulate PSMA expression on PCa cells (Supplemental Figure 1G), and synergize with $\left[{ }^{177} \mathrm{Lu}\right] \mathrm{Lu}$-DOTATATE in murine neuroendocrine tumors $(41,42)$.

\section{CONCLUSION}

Causes of RLT resistance are not well understood, respresenting a major barrier to urgently needed, more effective RLT approaches. While the generalizability of our findings is limited by use of a single immunocompromised mouse model, our study identifies tumor cell intrinsic mechanisms that might allow PCa cells to survive RLT, and key-effectors of these mechanisms that represent attractive targets for synergistic RLT-combination therapies (e.g., DDR/RSR, AR, MYC, PI3K/AKT/mTOR pathways). In ongoing studies, we are exploring these combination therapies with the aim to provide rationales for clinical studies. Association of PSMA-RLT (43) and identified key-effectors with tumor immunogenicity, including the upregulation of PD-L1 on tumor cells by radiation-induced ATR activation (44), supports the combination of RLT with DDR/RSR inhibitors and immunotherapies. Lastly, our data advocate for the systematic molecular profiling of PCa patients eligible for RLT with the goal to identify determinants and predictors of RLT-responsiveness that will guide patient stratification and the selection of RLT combination therapies. 
Conflict of Interest Statement: JC, CGR: co-founders and hold equity in Sofie Biosciences, Trethera Therapeutics. Intellectual property has been patented by UCLA and licensed to Sofie Biosciences, Trethera Therapeutics, but was not used in the current study. JCa: consulting activities outside of the submitted work for Advanced Accelerator Applications, Blue Earth Diagnostics, Janssen, Curium Pharma, GE Healthcare, Progenics, Radiomedix, Telix. WPF: consultant for Endocyte, BTG, received fees from RadioMedix, Bayer outside of the submitted work. No other potential conflict of interest relevant to this article was reported.

\section{Acknowledgments}

We thank Liu Wei, Larry Pang, Joel Almajano, Chloe Cheng, and Mark Girgis for their excellent assistance.

\section{Key Points}

Question: Which signaling mechanisms mediate resistance to PSMA-RLT, and could be targeted to increase RLT efficacy?

Pertinent Findings: PSMA-RLT induced alterations in the prostate cancer proteome/phosphoproteome, including deregulation of compensatory DNA damage and replication stress response pathways. TP53-status impacted response to PSMA-RLT in vivo.

Implications for Patient Care: Our results elucidate mechanisms underlying RLT-resistance and facilitate development of rational synergistic combination therapies. 


\section{References}

1. Hofman MS, Violet J, Hicks RJ, et al. [Lu-177]-PSMA-617 radionuclide treatment in patients with metastatic castration-resistant prostate cancer (LuPSMA trial): a single-centre, single-arm, phase 2 study. Lancet Oncol. 2018;19:825-833.

2. Kratochwil $\mathrm{C}$, Bruchertseifer $\mathrm{F}$, Rathke $\mathrm{H}$, et al. Targeted alpha-therapy of metastatic castrationresistant prostate cancer with 225Ac-PSMA-617: swimmer-plot analysis suggests efficacy regarding duration of tumor control. J Nucl Med. 2018;59:795-802.

3. Maier P, Hartmann L, Wenz F, Herskind C. Cellular pathways in response to ionizing radiation and their targetability for tumor radiosensitization. Int J Mol Sci. 2016;17:102.

4. Pilié PG, Tang C, Mills GB, Yap TA. State-of-the-art strategies for targeting the DNA damage response in cancer. Nat Rev Clin Oncol. 2019;16:81-104.

5.https://www.cbioportal.org/results/oncoprint?cancer_study_list=prad_mich\%2Cprad_su2c_2019\% 2Cprad_su2c_2015\%2Cprad_mpcproject_2018\&Z_SCORE_THRESHOLD=2.0\&RPPA_SCOR E_THRESHOLD $=2.0 \&$ data_priority=0\&profileFilter=0\&case_set_id=all\&gene_list=ATM\%250AA TR\%250ABRCA1\%250ABRCA2\%250ATP53\&geneset_list=\%20\&tab_index=tab_visualize\&Acti on=Submit. Accessed Sept 24, 2020.

6. Colletier PJ, Ashoori F, Cowen D, et al. Adenoviral-mediated p53 transgene expression sensitizes both wild-type and null p53 prostate cancer cells in vitro to radiation. Int J Radiat Oncol Biol Phys. 2000;48:1507-1512. 
7. Na R, Zheng SL, Han M, et al. Germline mutations in ATM and BRCA1/2 distinguish risk for lethal and indolent prostate cancer and are associated with early age at death. Eur Urol. 2017;71:740747.

8. van der Doelen MJ, Isaacsson Velho P, Slootbeek PHJ, et al. Impact of DNA damage repair defects on response to radium-223 and overall survival in metastatic castration-resistant prostate cancer. Eur J Cancer. 2020;136:16-24.

9. Kratochwil C, Giesel FL, Heussel CP, et al. Patients resistant against PSMA-targeting a-radiation therapy often harbor mutations in DNA damage-repair-associated genes. $J$ Nucl Med. 2020;61:683-688.

10. Platt RJ, Chen $S$, Zhou $Y$, et al. CRISPR-Cas9 knockin mice for genome editing and cancer modeling. Cell. 2014;159:440-455.

11. Fendler WP, Stuparu AD, Evans-Axelsson S, et al. Establishing (177)Lu-PSMA-617 radioligand therapy in a syngeneic model of murine prostate cancer. J Nucl Med. 2017;58:1786-1792.

12. Miederer M, Henriksen G, Alke A, et al. Preclinical evaluation of the alpha-particle generator nuclide 225Ac for somatostatin receptor radiotherapy of neuroendocrine tumors. Clin Cancer Res. 2008;14:3555-3561.

13. Current $\mathrm{K}$, Meyer $\mathrm{C}$, Magyar CE, et al. Investigating PSMA-targeted radioligand therapy efficacy as a function of cellular PSMA levels and intra-tumoral PSMA heterogeneity. Clin Cancer Res. 2020; 26:2946-2955. 
14. Abt ER, Rosser EW, Durst MA, et al. Metabolic modifier screen reveals secondary targets of protein kinase inhibitors within nucleotide metabolism. Cell Chem Biol. 2020; 27:197-205.e6.

15. Bonin $\mathrm{F}$, Molina M, Malet $\mathrm{C}$, et al. GATA3 is a master regulator of the transcriptional response to low-dose ionizing radiation in human keratinocytes. Bmc Genomics. 2009;10:417.

16. You A, Tong JK, Grozinger CM, Schreiber SL. CoREST is an integral component of the CoRESThuman histone deacetylase complex. Proc Natl Acad Sci U S A. 2001;98:1454-1458.

17. Yao K, Youn $\mathrm{H}, \mathrm{Gao} X$, et al. Casein kinase 2 inhibition attenuates androgen receptor function and cell proliferation in prostate cancer cells. Prostate. 2012;72:1423-1430.

18. Ha S, Ruoff R, Kahoud N, Franke TF, Logan SK. Androgen receptor levels are upregulated by Akt in prostate cancer. Endocr Relat Cancer. 2011;18:245-255.

19. Dagar M, Singh JP, Dagar G, Tyagi RK, Bagchi G. Phosphorylation of HSP90 by protein kinase A is essential for the nuclear translocation of androgen receptor. J Biol Chem. 2019;294:86998710 .

20. Wengner AM, Siemeister G, Lücking U, et al. The novel ATR inhibitor BAY 1895344 is efficacious as monotherapy and combined with DNA damage-inducing or repair-compromising therapies in preclinical cancer models. Mol Cancer Ther. 2020;19:26-38.

21. Mateo J, Porta N, Bianchini $\mathrm{D}$, et al. Olaparib in patients with metastatic castration-resistant prostate cancer with DNA repair gene aberrations (TOPARP-B): a multicentre, open-label, randomised, phase 2 trial. Lancet Oncol. 2020;21:162-174. 
22. Wickstroem K, Hagemann UB, Cruciani V, et al. Synergistic effect of a mesothelin-targeted (227)Th conjugate in combination with DNA damage response inhibitors in ovarian cancer xenograft models. J Nucl Med. 2019;60:1293-1300.

23. Wickstroem K, Karlsson J, Ellingsen C, et al. Synergistic effect of a HER2 targeted thorium-227 conjugate in combination with olaparib in a BRCA2 deficient xenograft model. Pharmaceuticals (Basel). 2019;12:155.

24. Cullinane C, Waldeck K, Kirby L, et al. Enhancing the anti-tumour activity of (177)Lu-DOTAoctreotate radionuclide therapy in somatostatin receptor-2 expressing tumour models by targeting PARP. Sci Rep. 2020;10:10196.

25. Xie H, Li C, Dang Q, Chang LS, Li L. Infiltrating mast cells increase prostate cancer chemotherapy and radiotherapy resistances via modulation of p38/p53/p21 and ATM signals. Oncotarget. 2016;7:1341-1353.

26. Kotler E, Segal E, Oren M. Functional characterization of the p53 "mutome". Mol Cell Oncol. 2018;5:e1511207.

27. Reinhardt HC, Aslanian AS, Lees JA, Yaffe MB. p53-deficient cells rely on ATM- and ATRmediated checkpoint signaling through the p38MAPK/MK2 pathway for survival after DNA damage. Cancer Cell. 2007;11:175-189.

28. Kumar D, Viberg J, Nilsson AK, Chabes A. Highly mutagenic and severely imbalanced dNTP pools can escape detection by the S-phase checkpoint. Nucleic Acids Res. 2010;38:3975-3983. 
29. Reichard P. Interactions between deoxyribonucleotide and DNA synthesis. Annu Rev Biochem. 1988;57:349-374.

30. Gioeli D, Ficarro SB, Kwiek JJ, et al. Androgen receptor phosphorylation - regulation and identification of the phosphorylation sites. J. Biol. Chem. 2002;277:29304-29314.

31. Polkinghorn WR, Parker JS, Lee MX, et al. Androgen receptor signaling regulates DNA repair in prostate cancers. Cancer Discov. 2013;3:1245-1253.

32. Shorning BY, Dass MS, Smalley MJ, Pearson HB. The PI3K-AKT-mTOR pathway and prostate cancer: At the crossroads of AR, MAPK, and WNT signaling. Int J Mol Sci. 2020;21:4507.

33. Chang L, Graham PH, Hao J, et al. PI3K/Akt/mTOR pathway inhibitors enhance radiosensitivity in radioresistant prostate cancer cells through inducing apoptosis, reducing autophagy, suppressing NHEJ and HR repair pathways. Cell Death Dis. 2014;5:e1437.

34. Dumont RA, Tamma M, Braun F, et al. Targeted radiotherapy of prostate cancer with a gastrinreleasing peptide receptor antagonist is effective as monotherapy and in combination with rapamycin. J Nucl Med. 2013;54:762-769.

35. Narayan V, Vapiwala N, Mick R, et al. Phase 1 Trial of everolimus and radiation therapy for salvage treatment of biochemical recurrence in prostate cancer patients following prostatectomy. Int J Radiat Oncol Biol Phys. 2017;97:355-361. 
36. Yao M, Rogers L, Suchowerska N, et al. Sensitization of prostate cancer to radiation therapy: Molecules and pathways to target. Radiother Oncol. 2018;128:283-300.

37. Nowak DG, Cho H, Herzka T, et al. MYC drives Pten/Trp53-deficient proliferation and metastasis due to IL6 secretion and AKT suppression via PHLPP2. Cancer Discov. 2015;5:636-651.

38. Hubbard GK, Mutton LN, Khalili M, et al. Combined MYC activation and Pten loss are sufficient to create genomic instability and lethal metastatic prostate cancer. Cancer Res. 2016;76:283-292.

39. Rebello RJ, Pearson RB, Hannan RD, Furic L. Therapeutic approaches targeting MYC-driven prostate cancer. Genes (Basel). 2017;8:71.

40. Kim SW, Hasanuzzaman M, Cho M, et al. Casein kinase 2 (CK2)-mediated phosphorylation of Hsp90 beta as a novel mechanism of rifampin-induced MDR1 expression. J. Biol. Chem. 2015;290:17029-17040.

41. Solit DB, Scher HI, Rosen N. Hsp90 as a therapeutic target in prostate cancer. Semin Oncol. 2003;30:709-716.

42. Lundsten S, Spiegelberg D, Stenerlow B, Nestor M. The HSP90 inhibitor onalespib potentiates 177LuDOTATATE therapy in neuroendocrine tumor cells. Int J Oncol. 2019;55:1287-1295.

43. Czernin J, Current K, Mona CE, et al. Immune-checkpoint blockade enhances ${ }^{225 A c-P S M A 617}$ efficacy in a mouse model of prostate cancer. J Nucl Med. 2020; [Epub ahead of print] 
44. Vendetti FP, Karukonda P, Clump DA, et al. ATR kinase inhibitor AZD6738 potentiates CD8+ T cell-dependent antitumor activity following radiation. J Clin Invest. 2018;128:3926-3940. 
Figures

A
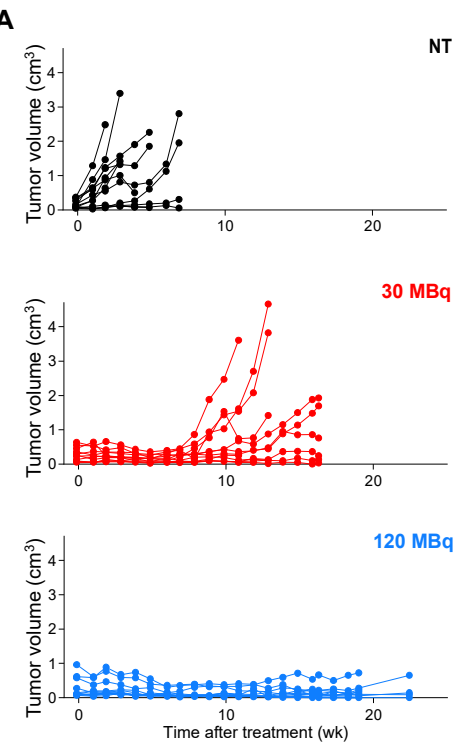

B
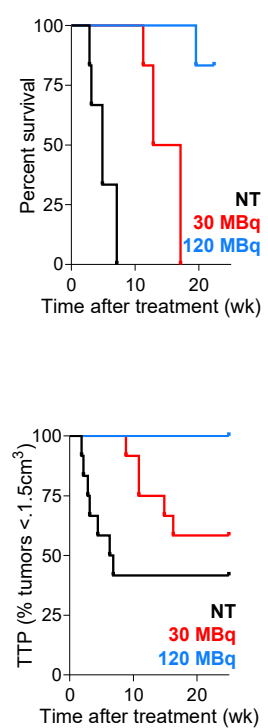

C

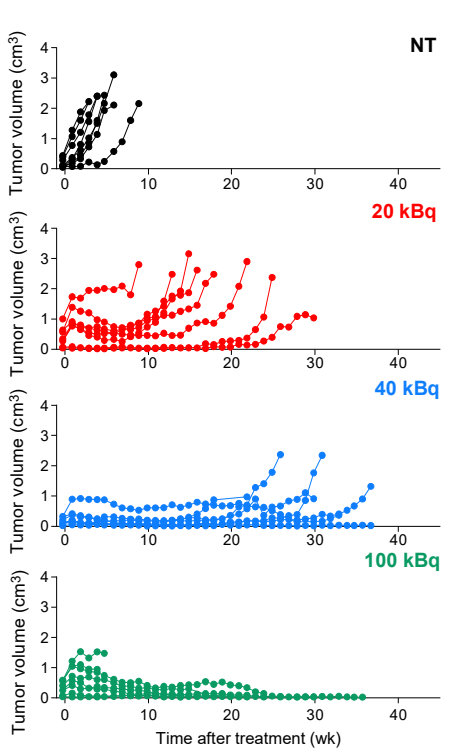

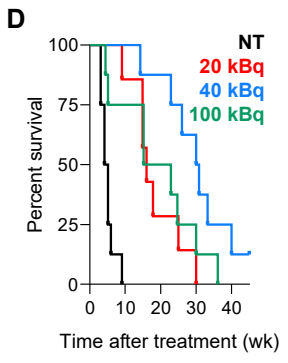

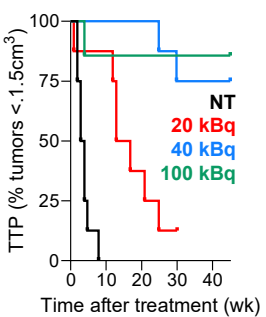

Figure 1. Optimizing treatment activities for $\left[{ }^{225} \mathrm{Ac}\right] \mathrm{Ac}-$ and $\left[{ }^{177}\right.$ Lu]Lu-PSMA-RLT. (A) Individual tumor growth curves following [177Lu]Lu-PSMA ( $n=12$ tumors, 6 mice/group; NT vs. RLT, $p \leq 0.0018$ [7wk]; $30 \mathrm{MBq}$ vs. $120 \mathrm{MBq}, p>0.99$ [7wk], $p=0.032$ [16wk]). (B) top: Survival: 4.8wk (NT), 15wk (30MBq), not reached (120MBq) ( $n=6$ mice/group: all $p \leq 0.001)$. bottom: TTP: 6.6wk (NT), not reached $(30 \mathrm{MBq}, 120 \mathrm{MBq})(\mathrm{n}=6$ mice/group; NT vs. $30 \mathrm{MBq}, p=0.153$; all other $\mathrm{p} \leq 0.014)$. (C) Individual tumor growth curves following $\left[{ }^{225} \mathrm{Ac}\right] \mathrm{Ac}-\mathrm{PSMA}-\mathrm{RLT}$ ( $\mathrm{n}=8$ mice/group; NT vs. RLT, p $\leq 0.027$ [6wk]; $20 \mathrm{kBq}$ vs. $40 \mathrm{kBq}$ or $100 \mathrm{kBq}, \mathrm{p}<0.023$ [15wk], 40kBq vs. $100 \mathrm{kBq}, \mathrm{p}>0.99)$. (D) top: Survival: 4.5wk (NT), 16wk (20kBq), 30wk (40kBq), 19wk (100kBq) (n=8 mice/group; p $\leq 0.0137$, except $40 \mathrm{kBq}$ vs. $100 \mathrm{kBq}, p=0.0783 ; 20 \mathrm{kBq}$ vs. $100 \mathrm{kBq}, p=0.6203)$. bottom: TTP: 3.5wk (NT), 15wk (20kBq), not reached $(40 \mathrm{kBq}, 100 \mathrm{kBq})(\mathrm{n}=8 \mathrm{mice} / \mathrm{group} ; \mathrm{p} \leq 0.0012$, except $40 \mathrm{kBq}$ vs. $100 \mathrm{kBq}, \mathrm{p}=0.679) . \mathrm{NT}=$ non-treated. 
A

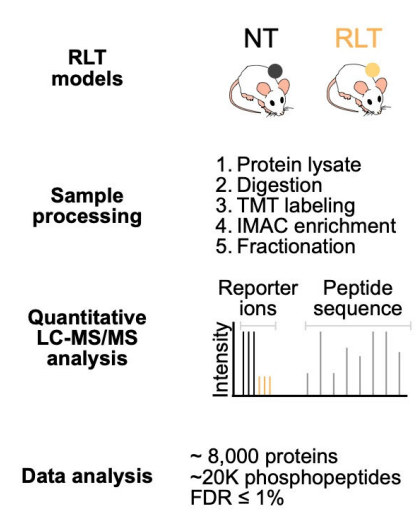

C

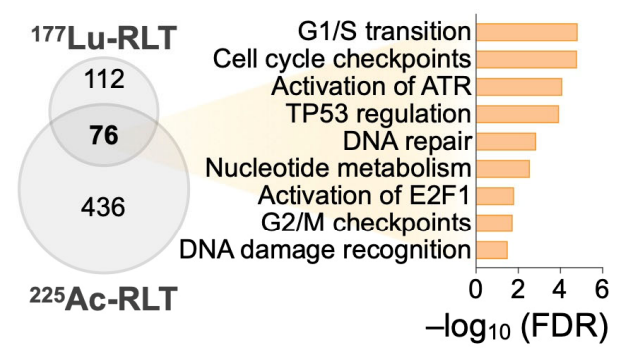

B

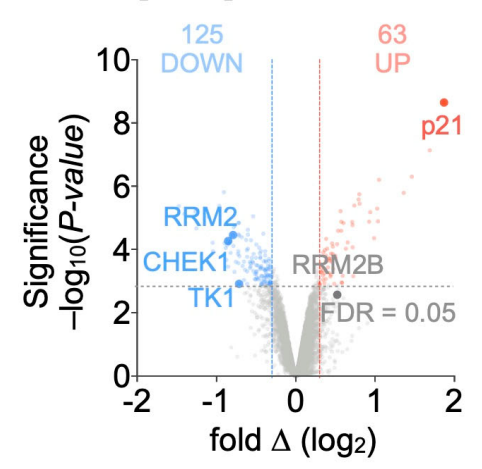

[225Ac]ACPSMA-RLT

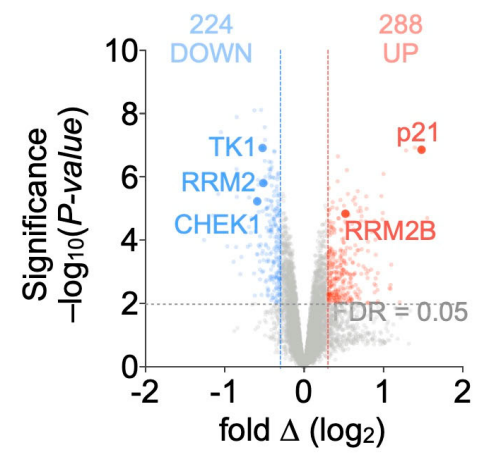

D down-regulating TFs up-regulating TFs
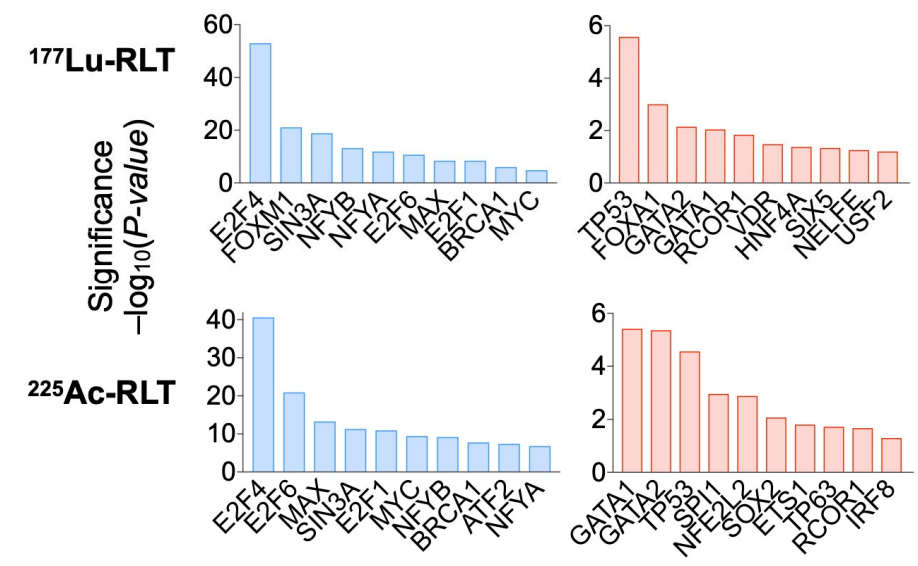

Figure 2. Proteomic analyses of PCa tumors reveal PSMA-RLT induced alterations. (A) Experimental workflow (also applies to Figure 3). (B) Volcano plots highlighting changes in protein levels relative to the untreated groups ( $n=188$ significant proteins for [ ${ }^{177}$ Lu]Lu-PSMA-RLT, 241 for $\left.\left[{ }^{225} \mathrm{Ac}\right] \mathrm{Ac}-\mathrm{PSMA}-\mathrm{RLT}\right)$. (C) Gene ontology analysis of the two datasets reveals commonly activated pathways. (D) Transcription factor enrichment analysis on differentially expressed proteins. Identified transcription factors have at least 4 associated targets. Graphs represent data from 3 tumors/group for [ $\left.{ }^{177} \mathrm{Lu}\right] \mathrm{Lu}-\mathrm{PSMA}-\mathrm{RLT}$ and 5 tumors/group for $\left[{ }^{225} \mathrm{Ac}\right] \mathrm{Ac}-\mathrm{PSMA}-\mathrm{RLT}$. 
A

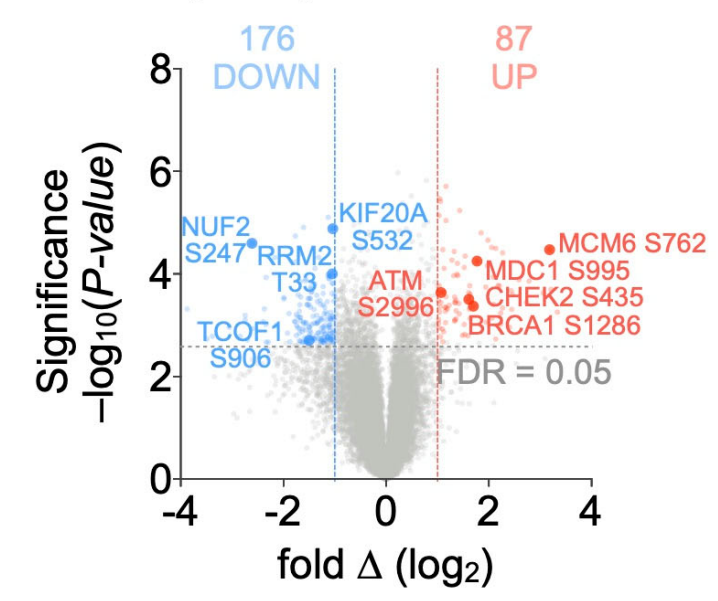

B

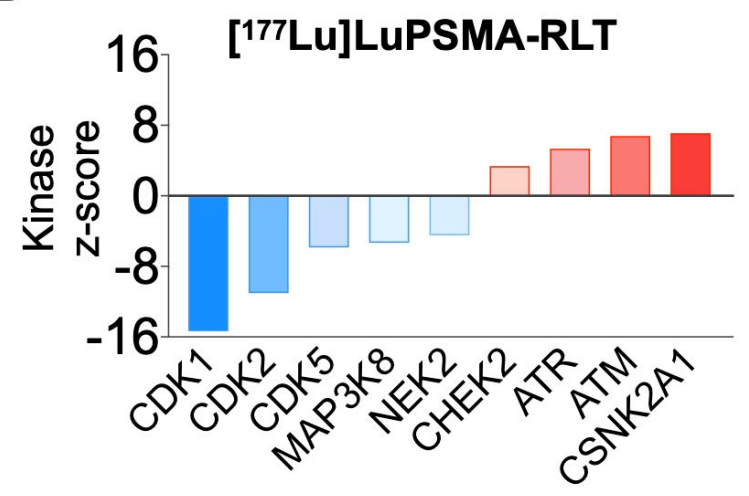

[225Ac]AcPSMA-RLT
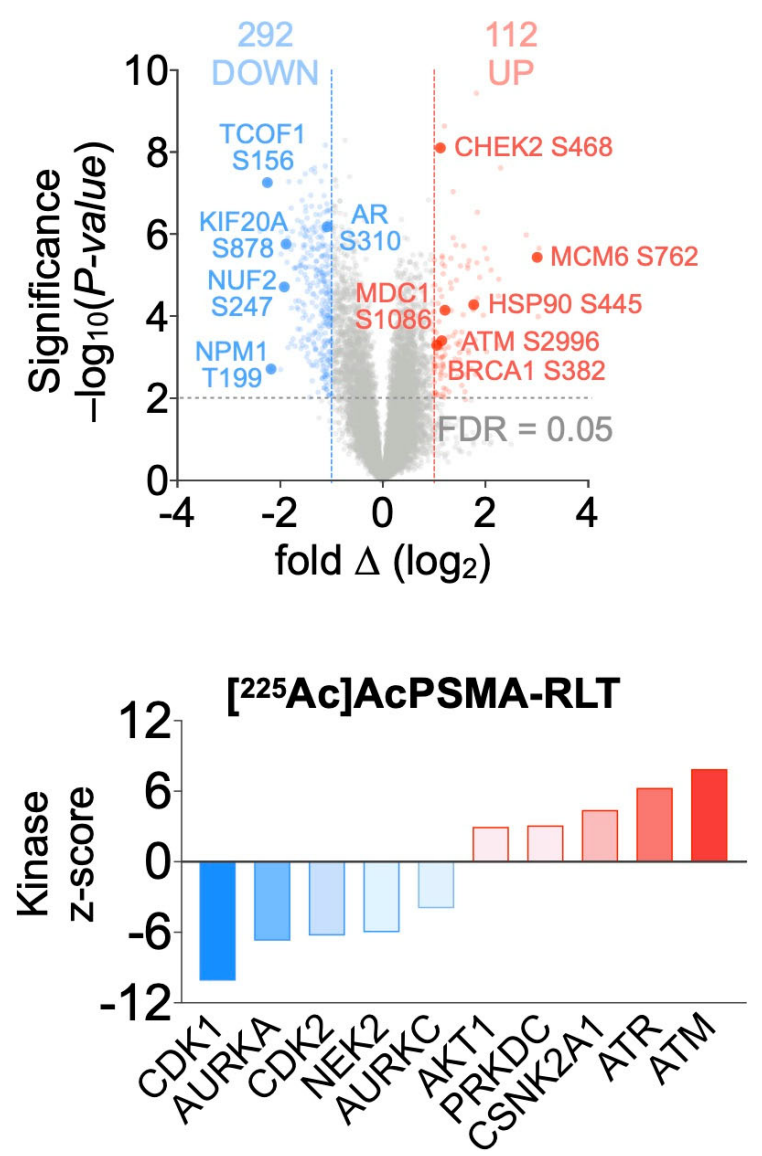

Figure 3. Phosphoproteomic analyses of PCa tumors reveal PSMA-RLT induced alterations. (A) Volcano plots of the identified phosphopeptides ( $n=512$ significant phosphopeptides for [ $\left.{ }^{177} \mathrm{Lu}\right] \mathrm{Lu}-\mathrm{PSMA}-\mathrm{RLT}$ and 405 for $\left.\left[{ }^{225} \mathrm{Ac}\right] \mathrm{Ac}-\mathrm{PSMA}-\mathrm{RLT}\right)$. (B) Kinasesubstrate enrichment analysis identifying kinases with at least 5 substrates. Graphs represent

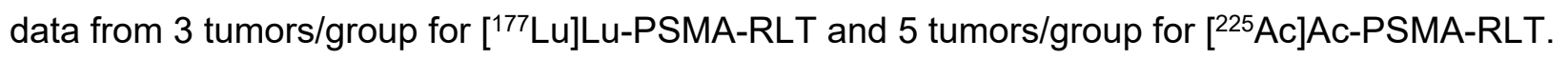




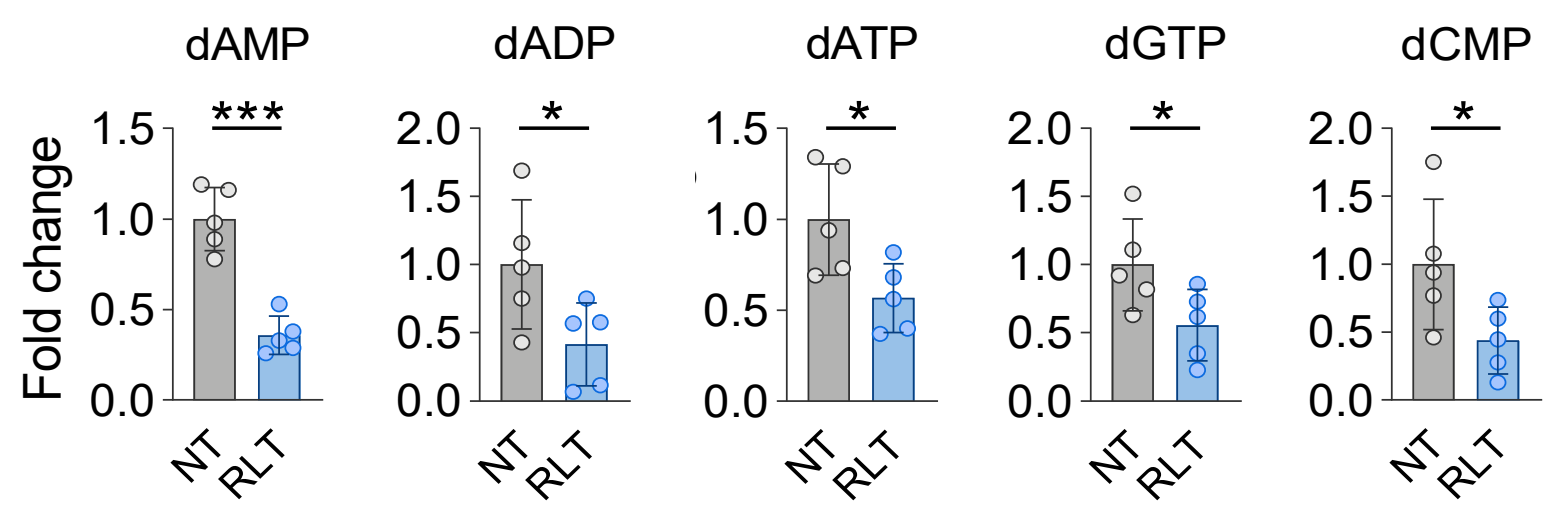

Figure 4. Nucleotide levels. Nucleotides are significantly altered by $\left[{ }^{225} \mathrm{Ac}\right] \mathrm{Ac}-\mathrm{PSMA}-\mathrm{RLT}$ compared to untreated controls $(p \leq 0.0499)$. Mean fold-change \pm standard deviation is shown, normalized to non-treated (NT) ( $n=5$ tumors/group). Significant differences are indicated by asterixes. dAMP - deoxyadenosine monophosphate, dADP - deoxyadenosine diphosphate, dATP - deoxyadenosine triphosphate, dGTP - deoxyguanosine triphosphate, dCMP - deoxycytidine monophosphate. 
A

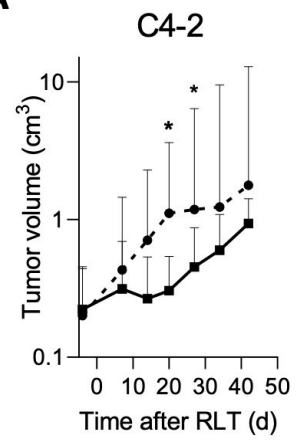

C4-2 TP53-1-

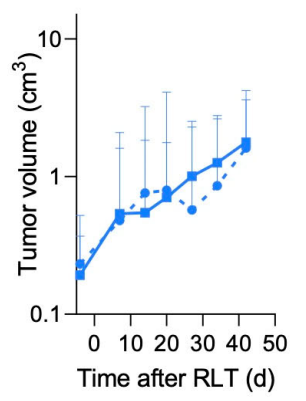

B

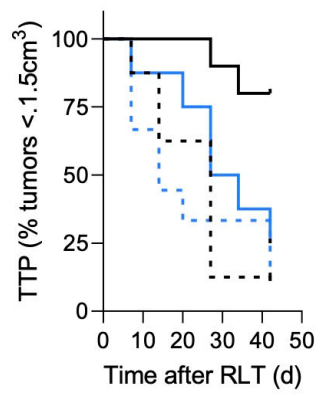

C

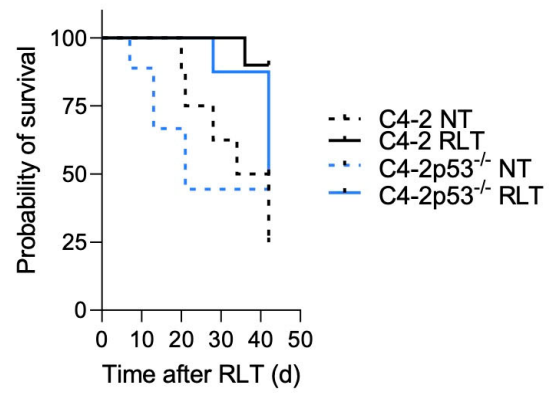

Figure 5. TP53-loss renders PCa resistant to PSMA-RLT. (A) Tumor growth of C4-2 (left) and C4-2 TP53-- (right) tumors treated with 15MBq [ $\left.{ }^{177} \mathrm{Lu}\right] \mathrm{Lu}-\mathrm{PSMA}-\mathrm{RLT}$. Geometric mean and 95\% confidence interval ( $n=8-10$ mice/group) are shown. Asterixes indicate significance. (B) TTP: C4-2: NT, 27d; RLT, undefined ( $p=0.0016)$; C4-2 TP53--.: NT 14d; RLT, 30.5d ( $p=0.3250)$. (C) Survival: C4-2: NT, 38d; RLT, undefined ( $p=0.0044)$; C4-2 TP53--: NT, 21d; RLT, 42d $(p=0.33939) . N T$ - no treatment. 


\section{Graphical abstract}

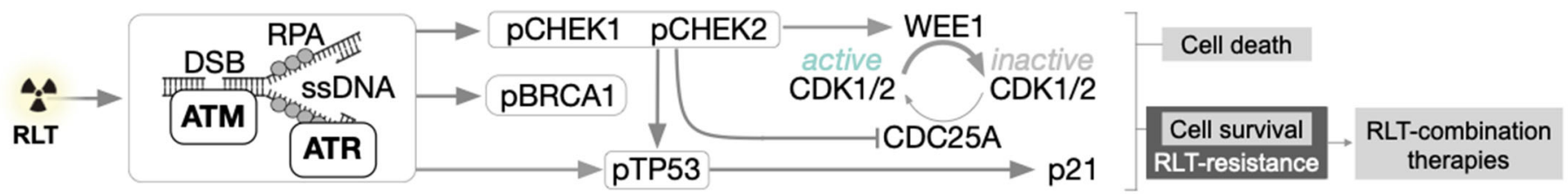

This study identifies signaling alterations induced by PSMA-RLT in prostate cancer that mediate resistance to RLT and could be exploited for synergistic RLT-combination therapies. 


\section{Mass Spectrometry}

\section{A. Protein extraction, digestion, labeling and pooling}

Tumors resected $48 \mathrm{hr}$ after treatment were snap-frozen in liquid nitrogen and homogenized using a bead mill (Omni Bead Ruptor 24) with 10 cycles of 30 s on 30 s off, power level 8, and chilled to $4^{\circ} \mathrm{C}$ with liquid nitrogen. One milliliter ice-cold, fresh lysis buffer (50 $\mathrm{mM}$ triethylammonium bicarbonate, $\mathrm{pH} 8.5,0.5 \%$ deoxycholate, $12 \mathrm{mM}$ sodium lauroyl sarcosine, protease inhibitor cocktail (Sigma) and phosphatase inhibitor cocktail containing $10 \mathrm{mM}$ sodium pyrophosphate, $50 \mathrm{mM}$ sodium fluoride, $1 \mathrm{mM}$ sodium orthovanadate, and $50 \mathrm{mM}$ betaglycerophosphate) was used for tumor homogenization. Tumor homogenates were centrifuged at $450 \mathrm{xg}$ for $5 \mathrm{~min}$ at $4^{\circ} \mathrm{C}$ and supernatants were sonicated for $10 \mathrm{~min}, 30 \mathrm{~s}$ on $30 \mathrm{~s}$ off, at $4^{\circ} \mathrm{C}$ using Bioruptor Pico (Diagenode) followed by heating at $95{ }^{\circ} \mathrm{C}$ for $5 \mathrm{~min}$. Protein concentrations of lysates were quantified by the BCA protein assay (Thermo Fisher Scientific), and $1 \mathrm{mg}$ total protein from each sample was carried through the rest of the sample preparation. Protein disulfides were reduced with $5 \mathrm{mM}$ dithiothreitol (final concentration) for $30 \mathrm{~min}$ at $37^{\circ} \mathrm{C}$, followed by alkylation with $10 \mathrm{mM}$ chloroacetamide (final concentration) for $30 \mathrm{~min}$ at room temperature in the dark. Excess alkylating agent was quenched by adding the same amount of dithiothreitol as in the previous step and incubating for $5 \mathrm{~min}$ at room temperature. Each sample was then diluted 1:5 using $50 \mathrm{mM}$ triethylammonium bicarbonate, $\mathrm{pH} 8.5$, containing $10 \mu \mathrm{g}$ trypsin (Promega) and digested at $37^{\circ} \mathrm{C}$ for $4 \mathrm{~h}$. A second aliquot of $10 \mu \mathrm{g}$ trypsin was spiked into the samples and digested overnight at $37^{\circ} \mathrm{C}$. The reaction was quenched and detergents were extracted with $1: 1$ (v:v) ethyl acetate containing $1 \%$ trifluoroacetic acid. The samples were vortexed vigorously and centrifuged at $16,000 \mathrm{xg}$ for $5 \mathrm{~min}$. The lower aqueous phase was transferred to new microcentrifuge tubes and dried using a SpeedVac vacuum concentrator. The samples were reconstituted in $2 \%$ acetonitrile with $0.1 \%$ trifluoroacetic acid, desalted on Oasis HLB $10 \mathrm{mg}$ cartridges (Waters), and dried using a SpeedVac. Samples were resuspended in 200 mM EPPS, 
$\mathrm{pH}$ 8.5, and peptide concentration was obtained using the Pierce Quantitative Colorimetric Peptide Assay. Four hundred microgram peptides were labeled with 10-plex TMT reagents at a 1:2 reagent : peptide concentration following the manufacturer's instruction, and dried by SpeedVac. Samples were reconstituted in $2 \%$ acetonitrile with $0.1 \%$ trifluoroacetic acid, and $1 \mu \mathrm{g}$ total peptides from each sample were pooled and desalted using SDB StageTips. The pooled sample is used to assess the TMT ratios for fine adjustments to the TMT reagent and sample volume to achieve better uniform ratios among the samples. One microgram of total peptide from this pooled sample was acquired via nanoLC-MS/MS on a QExactive Plus (Thermo) using a 3h gradient. The raw data were processed using Proteome Discoverer v2.2 (Thermo) (see E.). Each sample was normalized to the protein median fold change compared to the $126 \mathrm{~m} / \mathrm{z}$ TMT channel and $\sim 300 \mu \mathrm{g}$ of each sample was pooled accordingly. The pooled peptide sample was desalted on an Oasis HLB 200 mg cartridge (Waters) and dried by SpeedVac.

\section{B. Phosphopeptide enrichment by immobilized metal affinity chromatography}

For phosphopeptide enrichment using Fe-IMAC $3 \mathrm{mg}$ of dried peptides were solubilized in $900 \mu \mathrm{L}$ of phosphopeptide binding solution ( $80 \%$ acetonitrile and $0.1 \%$ TFA). One hundred fifty microliters peptide aliquots were mixed with $165 \mu \mathrm{L}$ of Fe-IMAC beads and incubated at room temperature for 30 min with shaking. The supernatant and all washes were collected, dried by SpeedVac, desalted on an Oasis HLB $200 \mathrm{mg}$ cartridge (Waters) and used for peptide fractionation and total protein quantification via nanoLC-MS/MS (see D.). The phosphopeptides were quickly eluted from beads with $100 \mu \mathrm{L}$ of phosphopeptide elution solution $(70 \%$ acetonitrile and $1 \%$ ammonium hydroxide), passed through C8 StageTip and acidified with $30 \mu \mathrm{L} 10 \%$ formic acid. The phosphopeptide eluents were dried by SpeedVac and desalted using SDB StageTips.

\section{Offline basic $\mathrm{pH}$ reverse phase liquid chromatography}

The complete phosphopeptide-enriched sample and $70 \mu \mathrm{g}$ of the non-phosphopeptide sample were each solubilized in $3 \mu \mathrm{L}$ buffer $\mathrm{A}$ (10 mM ammonium bicarbonate, $\mathrm{pH} 10$, and $2 \%$ 
acetonitrile) and separated on a Zorbax 300Extend-C18 column (3.5 $\mu \mathrm{m}$ particle size, $0.3 \mathrm{~mm} \times$ $150 \mathrm{~mm}$, Agilent) using an Agilent 1260 capillary pump and $\mu$ WPS autosampler equipped with an $8 \mu \mathrm{L}$ sample loop. Ninety-six fractions were collected with a 60 min gradient from $5-60 \%$ buffer B (90\% acetonitrile, $10 \mathrm{mM}$ ammonium bicarbonate, $\mathrm{pH} 10$, flow rate of $6 \mu \mathrm{L} / \mathrm{min}$ ) into pre-deposited $20 \mu \mathrm{L}$ of $5 \%$ formic acid. The samples were concatenated with an interval of 24 to form 24 final fractions (e.g., fractions 1, 25, 49, and 73 combined, fractions 2, 26, 50 and 74 combined, and so on). The concatenated fractions were desalted using SDB StageTips and dried by SpeedVac.

\section{Acidic pH reverse phase liquid chromatography coupled with tandem MS}

The dried peptide fractions for whole proteome analysis were reconstituted in $11 \mu \mathrm{L}$ of $2 \%$ acetonitrile and $0.15 \%$ formic acid. For phosphopeptide analysis, the dried peptide fractions were reconstituted in $6 \mathrm{uL}$ of $2 \%$ acetonitrile and $0.15 \%$ formic acid. Five microliters of sample were loaded on a laser-pulled reverse phase column $(150 \mu \mathrm{m} \times 20 \mathrm{~cm}, 1.8 \mu \mathrm{m}$ C18 resin with $0.5 \mathrm{~cm}$ of $5 \mu \mathrm{m} \mathrm{C4}$ resin at the laser-pulled end, Acutech Scientific, San Diego, CA) interfaced with an Eksigent 2D nanoLC, Phoenix S\&T dual column source, and QExactive Plus MS (Thermo). Peptides were eluted using 5-40\% buffer B gradient in 3h (buffer A: $2 \%$ acetonitrile, $0.15 \%$ formic acid; buffer B: $98 \%$ acetonitrile, $0.15 \%$ formic acid, flow rate of $0.5 \mu \mathrm{l} / \mathrm{min}$ ). The column was heated to $60^{\circ} \mathrm{C}$ by a butterfly portfolio heater (Phoenix S\&T) to reduce backpressure. The mass spectrometer was operated in data-dependent mode with a survey scan from 350-1500 m/z (70,000 resolution, $3 \times 106$ AGC target and 100 ms maximal ion time) and 10 MS/MS scans with starting fixed m/z of 100 (35,000 resolution, $2 \times 105$ AGC target, 120 ms maximal ion time, 32 normalized collision energy, $1.2 \mathrm{~m} / \mathrm{z}$ isolation window, and $30 \mathrm{~s}$ dynamic exclusion).

\section{E. Identification and quantitation of peptides using Proteome Discoverer v 2.2}

The acquired MS/MS raw files were searched by the Sequest algorithm against a forward and reverse target/decoy database to estimate FDR. The target protein database was downloaded from the Uniprot human database (reference and additional sequences, 93,320 
protein entries; downloaded in March 2018) and the decoy protein database was generated by reversing all target protein sequences. A contaminate protein database was included in the searches (244 protein entries; downloaded from MaxQuant 1.6.0.16). Spectra were searched with $\pm 10 \mathrm{ppm}$ for precursor ion and \pm 0.02 Da product ion mass tolerance, fully tryptic restriction, static mass shift for TMT-tagged N-terminus and lysine (+229.16293), carbamidomethylation on the cysteine $(+57.021)$, dynamic mass shift for oxidation of methionine $(+15.995)$, deamidation of asparagine and glutamine $(+0.984)$, acetylation of protein $\mathrm{N}$-terminus $(+42.011)$, phosphorylation of serine, threonine and tyrosine $(+79.96633$, only for phosphopeptide-enriched fractions), two maximal missed cleavages, three maximal modification sites, and the assignment of $\mathrm{b}$ and $\mathrm{y}$ ions. Putative peptide spectra matches were filtered by Percolator using $1 \%$ FDR. Post-translational modifications were site localized using ptmRS software. TMT reporter ions were quantified using the most confident centroid with reporter ion mass tolerance at $20 \mathrm{ppm}$.

\section{F. Differential expression analysis of the proteome and phosphoproteome}

Differential expression events were defined by identifying proteins/phosphopeptides with between-treatment variance significantly larger than within-replicate variance using one-way ANOVA (analysis of variance). Significantly altered proteins/phosphopeptides were filtered using Benjamini-Hochberg $(\mathrm{BH})$ procedure at 5\% FDR. All statistical analysis, principle component analysis, and unsupervised hierarchical clustering was performed using Python. Kinase-substrate enrichment analysis (KSEA) was performed using the KSEA App (https:// casecpb.shinyapps.io/ksea/). Briefly, the significantly-altered phosphopeptides were submitted and respective kinases were assigned using the PhosphositePlus database and NetworKin. Kinases were filtered with 5\% FDR ( $n=512$ significant phosphopeptides for ${ }^{177}$ Lu and 405 for $\left.{ }^{225} \mathrm{Ac}\right)$. 


\section{Supplemental Figures}

A

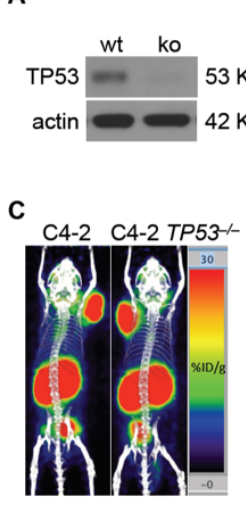

E

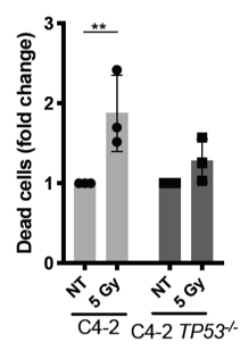

B

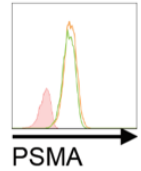

D

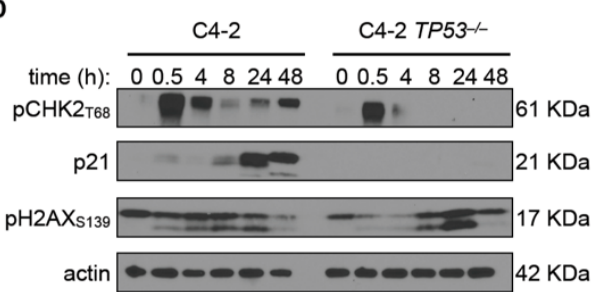

$\mathbf{F}$

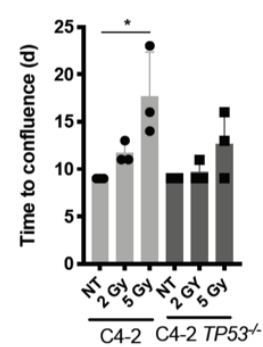

G

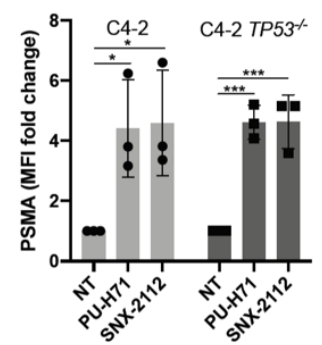

Supplemental Figure 1. Functional validation of C4-2 $\mathrm{TP}^{-/-}$cells. (a) Confirmation of Crispr/CAS9 mediated tp53 deletion in C4-2 cells by immunoblot (anti-p53, 7F5, dilution 1:1,000, \#2527; anti- $\beta$-actin, 8H10D10, 1:10,000, \#3700; Cell Signaling) $(n=1)$. (b, c) Comparable PSMA expression levels in wt (red) and ko (green) cells as assessed by flow cytometry using an antihPSMA-APC antibody (1:10 dilution, REA408, Miltenyi; BD LSR II flow cytometer). Filled red curve: unstained control $(n=1)(b)$, or by ${ }^{68} \mathrm{Ga}-\mathrm{PSMA}$ PET/CT images (1 representative mouse out of 5 is shown per group) (c). (d-f) Loss of TP53 increases resistance of C4-2 cells to radiation in vitro. (d) Immunoblot analysis of DNA damage response activation in vitro following irradiation with 5 Gy using antibodies detecting phospho-Chk2 (Thr68; C13C1, 1:1,000, \#2197), p21 (12D1; 1:1,000, \#2947), phospho-Histone H2A.X (Ser139; 20E3, 1:2,500, \#9718), and $\beta$-actin (8H10D10, 1:10,000, \#3700; all antibodies Cell Signaling) $(n=1)$. (e) Cell death following irradiation with 5 Gy was quantified by flow cytometry (propidium iodide). Data were normalized to untreated controls. (f) Cells were seeded on 6 -well plates $(100,000$ cells/well) and irradiated with 2 Gy or 5 Gy. The days it took cells to reach confluence was recorded. (g) Flow cytometric quantification of PSMA 
expression following treatment with HSP90 inhibitors for $72 \mathrm{~h}$. Data were normalized to untreated controls. In (e-g), mean \pm standard deviation are shown for 3 individual experiments. Significant differences are indicated by asterixes. ko - C4-2 TP53-- ; wt - C4-2 (parental); NT - not treated. 


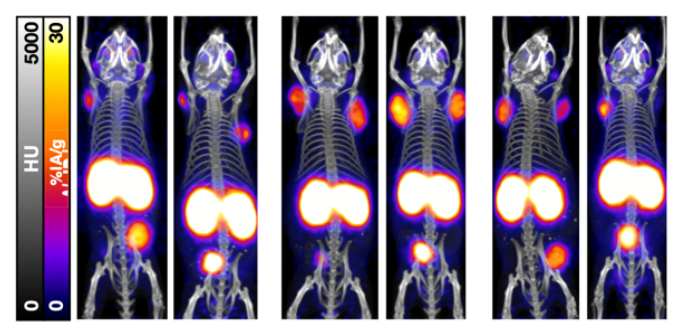

Supplemental Figure 2. C4-2 murine model validation. [ $\left.{ }^{68} \mathrm{Ga}\right] \mathrm{Ga}-\mathrm{PSMA}-11$ PET/CT confirms PSMA expression of C4-2 tumors one day before treatment. $\mathrm{HU}=$ Hounsfield Unit; \% $\mathrm{A} / \mathrm{g}=$ injected activity per gram 

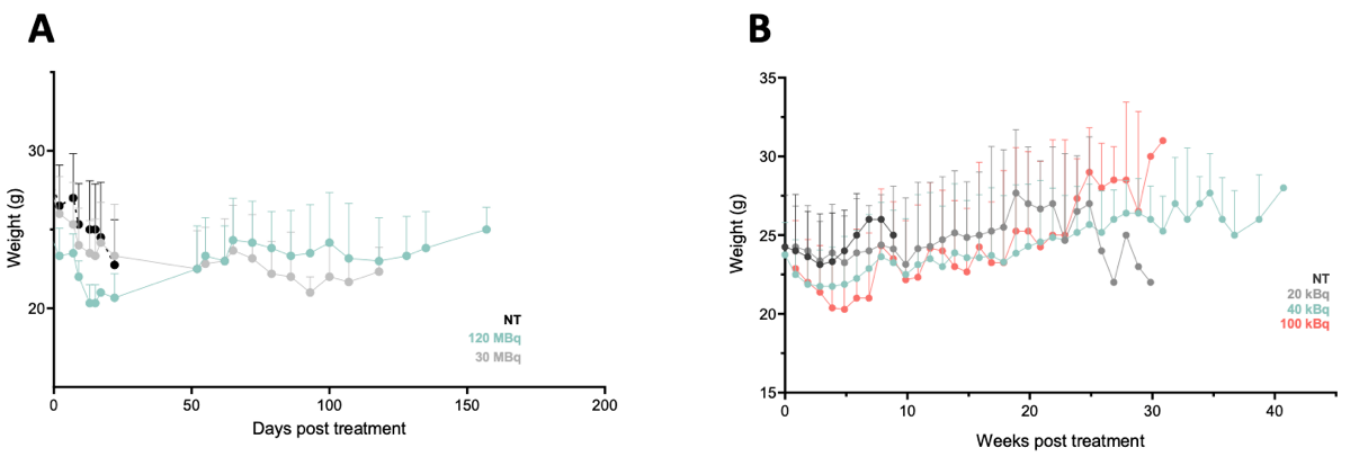

Supplemental Figure 3. Mouse weights. (A) $\left[{ }^{177}\right.$ Lu-]LuPSMA-RLT study ( $\mathrm{n}=6$ mice/group). (B) $\left[{ }^{225}\right.$ Ac]Ac-PSMA-RLT study ( $n=8$ mice/group). Data represent mean and standard deviation. 